Paediatric Psychology

\title{
Empathy and Moral Disengagement as Predictors of Bystander Roles in School Bullying
}

\author{
Zora Raboteg-Šarić ${ }^{1}$, Sanja Bartakovićn
}

${ }^{1}$ Ivo Pilar Institute of Social Sciences, Zagreb, Croatia, ${ }^{2}$ Izvor Kindergarten, Zagreb, Croatia

\begin{abstract}
Objective - The aims of this study were to explore bystander roles based on the Participant Role Questionnaire (PRQ) and to determine the contribution of affective empathy and moral disengagement in explaining bystander roles in bullying. Methods - The sample included 325 elementary school students aged 11-16 years old. Students' involvement in different bystander roles was assessed by subscales adapted from the PRQ, and their individual characteristics were measured by the Emotional Empathy Scale and the adapted version of the Moral Disengagement Scale. Results - The factor analysis revealed a five-factor structure of bystander role scales (assistant, reinforcer, defender, aggressive defender and outsider). The Outsider scale did not show satisfactory reliability. Boys had significantly higher scores on assisting the bully, whereas girls had significantly higher scores on defending the victim. Reinforcing the bully increased with students' age, while defending the victim decreased with age. Hierarchical regression analyses revealed that, after controlling for the effects of bystanders' gender and age, emotional empathy had significant positive effects on defender and aggressive defender roles, and a significant negative effect on the reinforcer role. Moral disengagement was a significant positive predictor of assistant, reinforcer and aggressive defender roles, and a negative predictor of the defender role. Conclusion - Bystanders' affective empathy and moral disengagement are important determinants of their behaviour in bullying situations. The validity of differentiating between the roles of assertive and aggressive defenders is confirmed.
\end{abstract}

Key Words: Bullying - Bystander Roles • Affective Empathy - Moral Disengagement - Adolescents.
(3). Bullying victims are more likely to develop symptoms of depression, internalizing (4) and psychosomatic problems (5), and are exposed to a higher risk of suicidal ideation (6). Long-term consequences include lower self-esteem, poor academic achievement, and poor psychosocial adjustment as adults (3). Children and adolescents who are bullies themselves are exposed to a higher risk of developing symptoms of anxiety and depression, social problems and rule-breaking 
behaviour (7), harmful alcohol use (4), subsequent offending, low job status and drug use (8).

Bullying has been most often examined from the victim and aggressor perspectives. In recent years, there is a growing body of research examining bullying as a group-level process, and the role of bystanders in the process. Studies of group dynamics in the classroom indicate that bullies are driven by a desire for dominance and they are often perceived as popular or powerful by their classmates. The mere presence of bystanders may induce bullies to violent behaviour in order to keep their reputation as powerful members of a group (2). Salmivalli et al. (9) identified four participant roles in the bullying process in a Finnish sample of sixth-graders, in addition to bullies or victims: assistants (follow or assist the bully), reinforcers (provide positive feedback to bullies), defenders (help or take sides with the victim), and outsiders (do nothing or stay out of bullying situations). Peers have been found to be present in most bullying incidents, but they rarely defend the victim. Observational data of bullying episodes on the school playground show that most of the time children stand by and passively observe violence $(54 \%)$ or actively engage on the bully's side (21\%), whereas only $25 \%$ of the time they actively defend the victim (10). Salmivalli et al. (9) classified $87 \%$ of the students based on peer-nominations. Approximately two-thirds of the students were bystanders of bullying episodes, while $12 \%$ were identified as victims and $8 \%$ as bullies. Defenders of the victim made up only $17 \%$ of the participants, while half of the participants actively or passively supported bullies either as reinforcers (19\%), assistants (7\%) or outsiders $(24 \%)$.

Although observers are present in most bullying situations, there is limited research about why people take different participant roles and why some children and youth ac- tively stand up against victimization (11). The research on defenders' characteristics and motivation has important practical implications for the prevention of school bullying and interventions aimed at reducing negative consequences of victimization. Victims who are defended by one or more students in the class show better adjustment (lower anxiety, lower depression, and higher self-esteem) and are less rejected by their peers than victims without defenders (12). Gender-related findings indicate that boys take the roles of assistants and reinforcers more often than girls, while girls act as defenders and outsiders more often than boys do $(9,13,14$, 15). Research on age differences has shown a growing trend in bullying among older elementary school students and younger high school students, and later bullying decreases with age (16). The results on age differences in bystander roles indicate that children and early adolescents are increasingly more likely to reinforce the bully (17) and less likely to defend the victim $(14,17)$. Tani et al. (18) demonstrated that other individual characteristics, such as personality traits, contribute to children's typical behaviour in bullying situations. Defenders of the victim exhibited the highest levels of friendliness (agreeableness) relative to their peers, while introversion and independence characterised outsiders. Both pro-bullies and victims demonstrated higher levels of emotional instability and lower levels of friendliness.

This paper focuses on examining the contributions of individual factors such as empathy and moral disengagement in explaining the behaviour of bystanders in school bullying situations. Empathy has been conceptualized and assessed as a multidimensional construct that includes both affective aspects, i.e. the tendency to experience emotions that are congruent with another's affective state, and cognitive aspects, such as the ability to understand other people's perspective (19). 
Empathy, especially its affective component, is consistently positively related to prosocial behaviour (20) and negatively related to aggressive and antisocial behaviours (21). The results of the research on the relationship between empathy and bullying are less consistent and may differ according to assessment methods, participants' gender or components of empathy. Recent literature reviews have shown that most studies found a negative correlation between cognitive and, in particular, affective empathy and overall bullying $(22,23)$. Less is known about the association of empathy with different bystander roles. Factor analyses of shortened adaptations of the original Participant Role Questionnaire (9) have rarely found a clear distinction between all participant roles, especially among the bully, the assistant and the reinforcer roles $(13,15,24)$. Similarly to bullies, children in pro-bullying roles lack empathic understanding for the victims $(25,26)$. The association between being an outsider and empathy remains unclear due to contradictory results, while defending the victim is consistently positively related to both affective and cognitive empathy (23). Opposing a powerful bully and his/her supporters requires not only empathy towards the victim but also adherence to moral standards that such behaviour is right. Studies have shown that children who are prone to violent behaviour toward their peers in real and virtual worlds have some similar characteristics such as a lack of empathy, a low level of morality, a lack of guilt, and a higher tendency for moral disengagement (27).

According to Bandura (28), moral behaviour (behaviour in accordance with internalized norms) is promoted by self-rewards, i.e. its positive consequences such as feelings of pride and self-worth. The same self-regulatory processes inhibit immoral behaviour because it is associated with negative consequences such as one's feelings of self-condemnation, guilt and shame. Moral disengagement mechanisms deactivate moral controls and allow people to justify their aggressive behaviour without experiencing negative consequences for self-image. Bandura (28) describes eight mechanisms of moral disengagement that are grouped into four categories. The first set of mechanisms refers to the cognitive restructuring of unacceptable behaviour, which is shown in a positive light through moral justification, euphemistic labelling, and favourable comparison with more harmful behaviour. The second set refers to diminishing or negating one's own responsibility for behaviour and its adverse consequences through the diffusion of responsibility or transfer of responsibility to someone else. The third category of moral disengagement mechanisms focuses on the adverse consequences of behaviour through minimizing, ignoring, or distorting them, and the fourth category includes humiliating the victim by dehumanizing the victim or blaming him/her for causing inappropriate behaviour. Bandura et al. (29) found that among the sample of 10 15 year-old participants, high moral disengagers are less prosocial and feel less guilty over detrimental conduct, and they engage in a higher level of interpersonal aggression. One meta-analysis of studies that included different samples of participants aged 8-18 years old found a positive overall effect of moral disengagement on aggressive behaviour and bullying, these associations being stronger in adolescents than in children (30).

The link between moral disengagement and participant roles in school bullying has been investigated in several studies. The tendency for moral disengagement has been positively related to bullying behaviour (31, $32,33)$, and pro-bullying roles $(31,34)$. Similarly to bullies, children in pro-bullying roles also have attitudes that are more approving of bullying (14). Outsiders' and defenders' behaviours are associated with lower levels of moral disengagement $(31,34)$. 
Limited research has sought to examine concurrently the effects of empathy and moral disengagement on bullying. In addition, many of the issues relevant to the assessment of bullying have focused on measures that assess students who bully and different types of victims. The present study aims to extend empirical findings on the links between students' personal characteristics and their roles in bullying by examining the simultaneous effects of affective empathy and moral disengagement on bystanders' behaviour. Although there is a growing body of research on bullying in Croatia, to our knowledge there are no published studies using a participant role approach and adaptation of the assessment instrument developed by Salmivalli et al. (9). The objectives of the present study were to explore bystander roles based on the Participant Role Questionnaire and to determine the contribution of affective empathy and moral disengagement in explaining bystander roles in school bullying situations.

\section{Methods}

\section{Participants}

The study included 325 students (167 girls and 158 boys) from two primary schools in the city of Zagreb (Croatia). The students were between the ages of 11 and 16 years old $(\mathrm{M}=12.95, \mathrm{SD}=1.15)$ and they were attending classes from four grade levels: fifth $(\mathrm{N}=74)$, sixth $(\mathrm{N}=83)$, seventh $(\mathrm{N}=80)$ and eighth grade $(\mathrm{N}=88)$.

\section{Instruments}

Participant Role Questionnaire (PRQ). This study used a modified version of the PRQ to assess children's reports of their behaviour when witnessing bullying situations in school. The PRQ is the peer-report instrument that was first applied in a Finnish sample of sixth-graders (9). Children rated on a 3-point scale the frequency of their and their classmates' behaviour on 50 items that describe different forms of student behaviour in bullying situations. Based on the results of factor analysis, 48 items were assigned to five scales: a Bully Scale (initiative taking, leaderlike bullying behaviour), the Assistant Scale (following, rather than instigating the bullying), the Reinforcer Scale (acting in ways that incite the bully thus providing an "audience" for the bully), the Defender Scale (providing support to the victim, efforts to make other stop bullying), and the Outsider Scale (staying out of the bullying situations). The reliabilities of the scales were good (the alpha coefficients were greater than 0.80 for each scale). In this study, we included 40 of the original descriptors of bystanders' roles in bullying situations. These items were translated from English into Croatian and checked through processes of forward and backward translation. The items of the Bully Scale were excluded from the development of adapted scales of the PRQ. Prior to the administration of the participant role scales, students were read the definition of bullying, which was also provided in written instructions. The PRQ specifies bullying as "one child being exposed repeatedly to harassment and attacks from one or several other children; harassment and attacks may be, for example, shoving or hitting the other one, calling names or making jokes of him/her, leaving him/her outside the group, taking his/ her things, or any other behaviour meant to hurt the other one." (9, p. 4). Students evaluated on a three-point scale how often they behaved in the ways described by each of the items ( 0 = "never", 1 = "sometimes", 2 = "often"). The exploratory factor analysis revealed five factors that explained $44.2 \%$ of the variance: Defender, Assistant (of the bully), Reinforcer (of the bully), Aggressive Defender, and Outsider. Item analyses indicated that four adapted scales had moderate to strong 
internal consistency: the Assistant Scale (8 items, $\alpha=0.71$ ), the Reinforcer Scale (5 items, $\alpha=0.69$ ), the Aggressive Defender Scale (4 items, $\alpha=0.71$ ), and the Defender Scale (14 items, $\alpha=0.89$ ). The Outsider Scale was not considered for further analyses due to its low reliability ( 5 items, Cronbach $\alpha=0.50$ ). For all scales, scores were computed by averaging across items, with higher scores indicating a stronger endorsement of the construct.

The Emotional Empathy Scale (EES). This instrument consists of 19 items that assess the affective component of empathy (35). Items (e.g. "I feel sorry when I see someone being helpless") describe susceptibility to emotional contagion, feelings of concern and sympathy, and emotional responsiveness to others' experiences. Students rated each item on a 5-point scale with response options ranging from 1 ("does not describe me well") to 5 ("describes me very well"). Prior studies with fourteen-year-old adolescents have shown satisfactory psychometric properties of this scale (35). The exploratory factor analysis confirmed a one-factor solution $(36.3 \%$ of the variance explained), and the internal consistency in the current sample was high $(\alpha=0.90)$. The overall score was formed by averaging the students' scores on all items. A higher score indicates greater empathic responsiveness.

The Moral Disengagement Scale (MDS). The original MDS was constructed by Hymel et al. (32) who identified 18 items from a survey with eighth- to tenth-grade Italian students on school bullying. These items described attitudes toward peer violence and reflected four major categories of moral disengagement described by Bandura (28): cognitive restructuring (e.g. "Bullying is just a normal part of being a kid"), minimizing agency (e.g. "It's my responsibility to intervene or do something when I see bullying" - reverse scored), distortion of negative consequences (e.g. "Bullying gets kids to understand what is important to the group"), and blaming or dehumanizing the victim (e.g. "Some kids get bullied because they deserve it"). Student responses were indicated on a 4-point scale (NO, no, yes, YES). Factor analysis of the results did not confirm the four-factor structure and 13 items loading significantly on a single, reliable factor $(\alpha=0.81)$ were retained for the final version of the scale. In the current study, we used adapted version of the MDS. The 18-item scale was translated from English into Croatian and then back translated. Participants rated each item on a 4-point scale (from 1="strongly disagree" to $4=$ "strongly agree"). The exploratory factor analysis yielded a one-factor solution for this scale $(31.6 \%$ of the total variance explained) with 15 items having significant loadings on a single factor. The Cronbach $\alpha$ reliability coefficient for this measure is 0.82 . The overall score was formed as the average of the answers to 15 items, with two items reverse scored. Higher scores in this scale indicate pro-bullying attitudes and a stronger tendency for moral disengagement.

Socio-demographic data. Demographic data were also collected and included students' age (years), gender (coded as $0=$ "male" and 1="female") and grade (1="fifth", $2=$ "sixth", 3="seventh", 4="eighth").

\section{Procedure}

Once school authorities had agreed to let their students participate in the study, parents received letters which explained the goals and procedures of the study. They were asked to contact the school if they did not wish their child to take part in the study. All of the parents gave consent for their children to participate. Students were informed that their participation was on a voluntary basis and they were free to withdraw from the study at any time. Four students did not agree to participate in the study. Students completed 
the questionnaires in their classrooms during regular school hours, under the supervision of one of the researchers. Instructions were written in the questionnaire and given orally as well. Data were collected and coded anonymously. The majority of students completed the questionnaire in less than 30 minutes.

\section{Statistical Analysis}

Data analyses were organised around the study aims. First, exploratory factor analysis was used to examine the factor structure of a shortened version of the PRQ among the sample of Croatian students who participated in this study. Item analyses were conducted to check the reliability of the adapted scales. Factor analysis of other instruments described in the Methods section was also performed and Cronbach's alpha was used to examine internal consistency. Descriptive statistics were obtained for all variables and a series of independent t-tests examined potential gender differences in the degree of involvement in different participant roles during school bullying, moral disengagement and emotional empathy. Pearson's bivariate correlation coefficients were computed to assess associations between variables. Finally, hierarchical regression analyses were computed to examine whether empathy and moral disengagement were significant predictors of students' involvement in different participant roles while controlling for age and gender effects. Missing values on measures of study variables $(<6 \%)$ were replaced by mean values on items of the same domain. Data analyses were performed using IBM SPSS Statistics for Windows, Version 25.

\section{Results}

\section{Factor Structure of the Adapted Participant Role Scales}

In this study, we were interested to examine students' behaviour in school bullying situa- tions when they witness other students being bullied. Therefore, we adapted the original PRQ (9) by including only those items that describe bystanders' behaviour and students rated the frequency of their typical behaviour when someone was being bullied. The behavioural descriptors included items from four subscales (roles) of the PRQ: Defender (20 items, Outsider (7 items), Reinforcer (7 items), and Assistant (4 items). We also included two items of the PRQ (one for the reinforcer role and one for the assistant role) that did not have significant factor loadings in the original study. We first examined whether adolescents in the present sample differentiated different forms of bystanders' behaviour as distinguished in the PRQ. A principal-components analysis of 40 items revealed the presence of eleven components with eigenvalues greater than 1 , which accounted for $59.5 \%$ of the total variance. The scree plot pointed to the existence of five factors. After factor analysis forcing five factors was employed and factor loadings below 0.30 were ignored, a simple five-factor structure emerged with one large and one small defender factor as well as assistant, reinforcer and outsider factors. We deleted items with ambiguous meaning, i.e. one item from this set that did not have significant loadings on any of the factors ("I didn't even know about the bullying"), one item that loaded equally on two (outsider and reinforcer) factors ("I didn't do anything") and one item describing the defender's role with higher factor loading on an unintended (reinforcer) factor than on the defender factor (I said to the victim: "Don't care about them"). Finally, the remining 37 items were submitted to a principal component analysis with oblimin rotation. Initial analysis revealed nine components with eigenvalues greater than one and the scree plot indicated that a five-factor solution would fit the data. The analysis was run again forcing five factors. The Keyser-Meyer-Olkin 
measure of 0.84 indicated a high sampling adequacy and Bartlett's test of sphericity was significant $(\mathrm{P}<0.001)$, indicating that the factor model is appropriate. Table 1 shows the

\section{Table 1. Factor Loadings on Items of Bystander Role Scales (Oblimin Rotation)}

\begin{tabular}{|c|c|c|}
\hline Factor & Items & Loadings \\
\hline \multicolumn{3}{|c|}{ F1: Defender } \\
\hline D1 & Comforts the victim afterwards & 0.76 \\
\hline D2 & Comforts the victim in the bullying situations & 0.76 \\
\hline D3 & Tells the others to stop bullying & 0.70 \\
\hline $\mathrm{D} 4$ & Stays with the victim during the breaks & 0.65 \\
\hline D5 & Says to the others that bullying is stupid & 0.63 \\
\hline D6 & Tries to make the others stop bullying & 0.61 \\
\hline D7 & Goes to tell the teacher about the bullying & 0.61 \\
\hline D8 & Tries to arbitrate the differences by talking & 0.60 \\
\hline D9 & Is friends with the victim during leisure time & 0.58 \\
\hline D10 & Tells some adult about the bullying & 0.56 \\
\hline D11 & Fetches the teacher in charge & 0.56 \\
\hline D12 & Fetches people to come and help the victim & 0.56 \\
\hline D13 & Encourages the victim to tell the teacher about the bullying & 0.50 \\
\hline D14 & Tells the others that it doesn't pay to join in the bullying & 0.40 \\
\hline \multicolumn{3}{|c|}{ F2: Assistant } \\
\hline A1 & Joins in the bullying, when someone else has started it & 0.64 \\
\hline $\mathrm{A} 2$ & Joins in the bullying when the others tell him/her to do so & 0.63 \\
\hline A 3 & Says to the bully: "Show him/her!" & 0.62 \\
\hline A 4 & Assists the bully & 0.59 \\
\hline A 5 & Catches the victim & 0.55 \\
\hline A 6 & Incites the bully by shouting & 0.52 \\
\hline A 7 & Holds the victim, when he/she is harassed & 0.46 \\
\hline A 8 & Says to the others: "Come to see, someone is being harassed there!" & 0.34 \\
\hline \multicolumn{3}{|c|}{ F3: Outsider } \\
\hline $\mathrm{O} 1$ & Stays outside the situation & 0.66 \\
\hline $\mathrm{O} 2$ & Pretends not to notice what is happening & 0.62 \\
\hline O3 & Doesn't take sides with anyone & 0.55 \\
\hline $\mathrm{O} 4$ & Is not usually present & 0.55 \\
\hline O5 & Goes away from the spot & 0.41 \\
\hline \multicolumn{3}{|c|}{ F4: Aggressive Defender } \\
\hline $\mathrm{AD} 1$ & Says to the others that the bully is stupid & 0.73 \\
\hline $\mathrm{AD} 2$ & Calls the bullies names in order to defend the victim & 0.69 \\
\hline AD3 & Attacks the bully in order to defend the victim & 0.68 \\
\hline $\mathrm{AD} 4$ & Takes revenge on the bully for the victim & 0.57 \\
\hline AD5 & Threatens to tell the teacher, if the others don't stop bullying & 0.41 \\
\hline \multicolumn{3}{|c|}{ F5: Reinforcer } \\
\hline $\mathrm{R} 1$ & Stays near and looks & 0.62 \\
\hline $\mathrm{R} 2$ & Is usually present, even if not doing anything & 0.61 \\
\hline $\mathrm{R} 3$ & Comes around to see the situation & 0.59 \\
\hline $\mathrm{R} 4$ & Giggles & 0.57 \\
\hline R5 & Laughs & 0.51 \\
\hline
\end{tabular}


factor loadings of the rotated 37-item set. All items loaded on one of the five factors and accounted for $44.2 \%$ of the variance. There were no items that cross-loaded onto any other factor above 0.30 . The eigen-values associated with these factors (F1 to F5) were $6.91,4.32,2.03,1.68$, and 1.46, respectively.

The first factor was labelled the Defender (of the victim) role and included 14 defender items of the PRQ that appropriately loaded on this factor. The second factor corresponded to the Assistant (of the bully) role and included four Assistant items as well as three items from the Reinforcer scale of the original instrument (A3, A6 and A8) that describe direct involvement in supporting the bully. In addition, one item that describes active involvement in bullying (A2) was dropped from the PRQ, while in our sample this item loaded on the Assistant factor. The third factor was labelled the Outsider and included items from the same scale of the PRQ. The fifth factor was labelled the Reinforcer (of the bully) and included four items from the same scale of the PRQ and one descriptor of the reinforcer role that was dropped from the original scale (R1). These items describe inciting the behaviour of the bully in indirect ways by being present and paying attention to the bullying situation or laughing. These four factors of the shorter version of the PRQ that was applied in our study correspond to the four bystanders' roles of the original instrument. However, five items from the original Defender Scale had significant loadings on the separate (fourth) factor, which was labelled the Aggressive Defender (of the victim). These items describe less constructive ways of dealing with bullying situations in order to support the victim, which include verbal and physical aggression and revengeful behaviour directed towards the bullies.

Individual mean responses were calculated for each factor and item analyses were performed to check for the reliabilities of the adapted bystander role scales. One item was dropped from the Aggressive Defender Scale (AD5) since item analysis showed that its removal would improve the reliability of this scale. The Outsider Scale had low internal consistency (Cronbach's $\alpha=0.50$ ) and was not included in further data analysis. Alpha coefficients for other bystander role scales were acceptable, indicating moderate to high reliability (Reinforcer $=0.69$, Assistant $=0.71, \mathrm{Ag}$ gressive Defender=0.71, Defender=0.89).

\section{Descriptive Statistics, Gender Differences and Intercorrelations Between the Study Variables}

Mean scores on bystander role scales show that students reported being more frequently involved as defenders of the victim $(M=1.10$, $\mathrm{SD}=0.44)$ than they reported for reinforcing the bully $(\mathrm{M}=0.79, \mathrm{SD}=0.40)$ or taking the role of the aggressive defender of the victim $(\mathrm{M}=0.58, \mathrm{SD}=0.47)$, while the mean self-reported involvement as assistants of the bully was the lowest $(\mathrm{M}=0.18, \mathrm{SD}=0.25)$. Students tend to report moderately high levels of emotional empathy $(\mathrm{M}=3.72, \mathrm{SD}=0.66)$, while the mean score on the Moral Disengagement Scale indicates moderately low tendency for moral disengagement in bullying situations $(\mathrm{M}=1.92, \mathrm{SD}=0.40)$.

Pearson's intercorrelation coefficients between the study variables are given in Table 2. Correlations among the adapted PRQ scales provided some evidence of their construct validity. The Assistant, Reinforcer and Defender scales were significantly related in a predictable pattern. Expectedly, the strongest positive correlation was found between the two self-reported behavioural measures on pro-bullying scales, the Assistant and Reinforcer scales. The Defender and Reinforcer scales were significantly negatively related, and a small negative correlation was also found between the Defender and Assistant scores. Students' scores on the Aggressive De- 
Table 2. Correlations between Bystander Role, Emotional Empathy and Moral Disengagement Scales

\begin{tabular}{llllllll}
\hline Scale & & 1 & 2 & 3 & 4 & 5 & 6 \\
\hline 1 & Assistant & 1 & $0.47^{\dagger}$ & $0.35^{\dagger}$ & $-0.13^{*}$ & $-0.16^{\dagger}$ & $0.42^{\dagger}$ \\
\hline 2 & Reinforcer & - & 1 & $0.30^{\dagger}$ & $-0.32^{\dagger}$ & $-0.21^{\dagger}$ & $0.46^{\dagger}$ \\
\hline 3 & Aggressive defender & - & - & 1 & $0.14^{*}$ & 0.05 & $0.32^{\dagger}$ \\
\hline 4 & Defender & - & - & - & 1 & $0.50^{\dagger}$ & $-0.29^{\dagger}$ \\
\hline 5 & Emotional empathy & - & - & - & - & 1 & $-0.26^{\dagger}$ \\
\hline 6 & Moral disengagement & - & - & - & - & - & 1 \\
\hline
\end{tabular}

${ }^{*} \mathrm{P}<0.05 ;{ }^{\dagger} \mathrm{P}<0.01$.

fender scale were significantly positively associated with Assistant and Reinforcer scales. These scales measure similar forms of bullying behaviours that are directed either to the victim or to the bully. In contrast, a small significant positive correlation emerged between the Aggressive Defender and Defender scales. Both scales measure behaviours directed to support the victim while using either aggressive or assertive ways of helping the victim. Correlations with empathy and moral disengagement measures further confirm the independency of the Defender and the Aggressive Defender constructs. Scores on the Defender Scale were significantly positively associated with emotional empathy and negatively associated with moral disengagement, while Aggressive Defender scores were positively related to moral disengagement scores and were not associated with emotional empathy. The assistant and reinforcer roles were significantly positively associated with moral disengagement scores and negatively associated with empathy scores.

With respect to behaviour in bullying situations, two variables showed significant correlations with students' age. Reinforcing the bully increased with age $(\mathrm{r}=0.18, \mathrm{P}<0.01)$, while defending the victim decreased with age $(r=-0.15, P<0.01)$. Assistant $(r=0.03$, $P>0.05)$ and aggressive defender roles $(\mathrm{r}=0.10, \mathrm{P}>0.05)$ were not associated with students' age. Older students showed a some-

\begin{tabular}{lllllll} 
Table 3. Gender Differences on Study Variables \\
\cline { 1 - 5 } Variable & \multicolumn{3}{c}{ Boys $(\mathrm{N}=158)$} & \multicolumn{2}{c}{ Girls $(\mathrm{N}=167)$} & \\
\cline { 2 - 5 } & $\mathrm{M}$ & $\mathrm{SD}$ & $\mathrm{M}$ & $\mathrm{SD}$ & \\
\hline Assistant & 0.25 & 0.29 & 0.12 & 0.18 & $4.86^{\dagger}$ \\
\hline Reinforcer & 0.83 & 0.42 & 0.76 & 0.38 & 1.55 \\
\hline $\begin{array}{l}\text { Aggressive } \\
\text { defender }\end{array}$ & 0.62 & 0.49 & 0.55 & 0.44 & 1.35 \\
\hline Defender & 1.04 & 0.47 & 1.16 & 0.40 & $-2.40^{*}$ \\
\hline $\begin{array}{l}\text { Emotional } \\
\text { empathy }\end{array}$ & 3.55 & 0.70 & 3.88 & 0.56 & $-4.77^{\dagger}$ \\
\hline $\begin{array}{l}\text { Moral } \\
\text { disengagement }\end{array}$ & 2.01 & 0.42 & 1.82 & 0.35 & $4.33^{\dagger}$ \\
\hline
\end{tabular}

${ }^{*} \mathrm{P}<0.05 ;{ }^{\dagger} \mathrm{P}<0.01$

what higher tendency for moral disengagement in bullying situations $(\mathrm{r}=0.11, \mathrm{P}=0.05)$, while empathic responsiveness was not related to students' age $(\mathrm{r}=-0.04, \mathrm{P}>0.05)$.

Independent sample $\mathrm{t}$-tests examined possible significant gender differences on the variables under study. As Table 3 shows, boys had significantly higher mean scores on assisting the bully while girls had significantly higher scores on defending the victim. There were no significant gender differences for the reinforcer and aggressive defender roles. Boys and girls differed significantly on the constructs of moral disengagement and emotional empathy. Boys reported more moral disengagement while girls reported higher empathic responsiveness. 


\section{Hierarchical Multiple Regression Analyses}

To examine the relative contribution of the predictor variables to the explanation of students' typical behaviour when witnessing bullying situations, four hierarchical multiple regression analyses were conducted (Enter method) with assistant, reinforcer, aggressive defender, and defender roles as criterion variables. Students' gender and age were entered into the regression equation as a block in the first step of analysis. To examine the effects of moral disengagement and empathy independently of the students' gender and age, these variables were entered as a block in the second step of analysis.

As summarised in Table 4, socio-demographic variables showed a significant, but relatively small, contribution to the explanation of variance in assistant $(7 \%)$, reinforcer $(4 \%)$ and defender (4\%) roles at the first step of a hierarchical regression analysis. The inspection of beta coefficients shows that gender was the single predictor of the assistant role, indicating that females were less involved in this role, while age was the single predictor of the reinforcer role, with older students being more frequently involved in reinforcing the bully. Both socio-demographic variables significantly predicted the role of the defender, indicating that females and younger students were more involved in defending the victims. Adding empathy and moral disengagement to the model led to a significant improvement of the total variance explained for the assistant (14\%), the reinforcer $(20 \%)$, the aggressive defender (11\%) and the defender $(25 \%)$ roles. In addition to gender and age, there was a unique contribution of moral disengagement for each of the four bystander roles, and a unique contribution of emotional empathy for three bystander roles. The inspection of beta coefficients revealed that emotional empathy had independent positive effects on the defender and aggressive defender roles and a small negative effect on the reinforcer role. Empathy was not associated with aggressive defending,

\begin{tabular}{|c|c|c|c|c|}
\hline \multirow{2}{*}{ Variables } & Assistant & Reinforcer & Aggressive defender & Defender \\
\hline & $\beta$ & $\beta$ & $\beta$ & $\beta$ \\
\hline \multicolumn{5}{|l|}{ Step1 } \\
\hline Gender & $-0.26^{* *}$ & -0.09 & -0.08 & $0.13^{*}$ \\
\hline Age & 0.03 & $0.18^{* *}$ & 0.10 & $-0.15^{* *}$ \\
\hline $\mathrm{R}^{2}$ & $0.07^{* *}$ & $0.04^{* *}$ & 0.02 & $0.04^{* *}$ \\
\hline $\mathrm{F}(2,322)$ & $11.93^{* *}$ & $6.65^{* *}$ & 2.54 & $6.46^{* *}$ \\
\hline \multicolumn{5}{|l|}{ Step 2} \\
\hline Gender & $-0.17^{* *}$ & 0.04 & -0.03 & -0.03 \\
\hline Age & -0.04 & $0.13^{* *}$ & 0.07 & $-0.11^{*}$ \\
\hline Emotional empathy & -0.02 & $-0.10^{*}$ & $0.15^{* *}$ & $0.46^{* *}$ \\
\hline Moral disengagement & $0.38^{* *}$ & $0.43^{* *}$ & $0.34^{* *}$ & $-0.17^{* *}$ \\
\hline$\Delta \mathrm{R}^{2}$ & $0.14^{* *}$ & $0.20^{* *}$ & $0.11^{* *}$ & $0.25^{* *}$ \\
\hline$\Delta \mathrm{F}(2,320)$ & $27.61^{* *}$ & $42.68^{* *}$ & $19.80^{* *}$ & $57.64^{* *}$ \\
\hline $\mathrm{R}^{2}$ & $0.21^{* *}$ & $0.24^{* *}$ & $0.12^{* *}$ & $0.29^{* *}$ \\
\hline $\mathrm{F}(4,320)$ & $20.75^{* *}$ & $25.53^{* *}$ & $11.32^{* *}$ & $33.19^{* *}$ \\
\hline
\end{tabular}

${ }^{*} \mathrm{P}<0.05 ;{ }^{*} \mathrm{P}<0.01 ; \beta=$ The standardized beta coefficient; $\mathrm{R}^{2}=$ The coefficient of multiple determination; $\Delta \mathrm{R}^{2}=$ The change in $\mathrm{R}^{2} ; \Delta \mathrm{F}=\mathrm{F}$ for change in $\mathrm{R}^{2}$. 
but when entered in the regression equation with moral disengagement this variable acted as a suppressor and enhanced the prediction of aggressive defender criterion. Emotional empathy was not an independent significant predictor of the assistant role. Partial correlation analysis showed that negative association between empathy and assisting the bully was no longer significant when gender differences in these variables were controlled. Moral disengagement had independent positive effects on the reinforcer, assistant and aggressive defender roles, indicating that pro-bullying attitudes were associated with greater involvement in assisting and reinforcing the bully and with a greater involvement in aggressive behaviours in defending the victim. Moral disengagement had a smaller, but significant negative effect on the defender role, indicating that pro-bullying attitudes were related to less frequent involvement in defending the victim. Gender was significantly related to the defender role at the first step of a hierarchical regression analysis, but its association with the defender behaviour was not significant when emotional empathy and moral disengagement were entered into the regression equation at the second step of the analysis. Partial correlational analysis indicated that gender differences in the defender role were mediated by differences in both affective empathy and moral disengagement between boys and girls. The final regression model explained a significant percentage of variance in all bystander roles. Overall, predictors included in this study explained the highest proportion of variance in the defender role $(29 \%)$, followed by reinforcer $(24 \%)$ and assistant $(21 \%)$ roles, and the lowest percentage of variance $(12 \%)$ in the aggressive defender role.

\section{Discussion}

The present study was undertaken in order to examine the role of bystanders' individual characteristics, such as emotional empathy and moral disengagement, in explaining their behaviour in school bullying situations. Due to the overall lack of empirical studies in Croatia that focus on participant roles in bullying, our first aim was to examine the validity of adapted scales of the PRQ (9) that assess bystander roles on a sample of 11 to 16 year-old Croatian adolescents. The factor analysis revealed a five-factor structure with four bystander roles as in the PRQ (i.e., Assistant, Reinforcer, Defender, and Outsider), and one separate small defender factor that was labelled "Aggressive Defender." Reinforcer, assistant and defender scores were significantly correlated and the pattern of intercorrelations among these role behaviours was similar to previous research (9). Moderate positive correlations were found between reinforcer and assistant scales that assess similar pro-bullying constructs, and smaller but significant negative correlations were found between both scales and defender scores. Contrary to this, aggressive defender scores were significantly positively related to reinforcer and assistant roles. The two defender scales were significantly positively associated, but this correlation was smaller than intercorrelations of aggressive defender scores with pro-bullying roles. To our knowledge, other studies that assess participant roles in bullying have not considered the role of the aggressive defender. Our findings are in accordance with the Hawkins et al. (36) observational study of children's interventions in bullying on the school playground. Peers were present during $88 \%$ of bullying episodes and intervened in only $19 \%$ of the episodes. In $47 \%$ of intervention episodes, defenders reacted aggressively. The majority of interveners chose to target the bully and they were more likely to choose aggressive strategies, whereas interventions directed at victims and bully-victims dyad were more likely to be assertive (non-aggressive). Poz- 
zolli and Gini (37) found that defenders are more likely than outsiders to use problemsolving strategies in response to observations of bullying. The behaviour of the defender, as identified in our study, is characterized with problem-solving strategies and assertive ways of conflict resolution in bullying situations.

Existing studies have used different adaptations of the PRQ, and comparison of their results is difficult due to methodological differences in assessing participant roles, items included in factor analytic procedures and the age of the participants (24). Most studies included items for all six roles (i.e. bully, victim and four bystander roles) and reduced the number of items, while some studies dropped the single victim-nomination item or included additional items for victimization. Studies with children in the United Kingdom (15), Italy (18), and Spain (24) found a unique factor that accounted for the Bully, Assistant, and Reinforcer scales, while defender and outsider items consistently loaded on separate dimensions. Other studies using peer-nomination (13) and selfrating scale format (26) for assessing different participant roles revealed that assistant and reinforcer items loaded on the same factor. The adapted version of the PRQ in our study allows us to discriminate between defenders who typically demonstrate either assertive or aggressive behaviours as well as to discriminate between assistant and reinforcer roles. Items that assess defenders' aggressive behaviours are omitted from final versions of shortened adaptations of the PRQ in other studies, while the adapted version in our study contains almost all items that measure bystander roles. The role of the outsider also appeared as a separate factor, but internal consistency of the Outsider scale was low. Future adaptations of bystander role scales in the Croatian cultural context should include more outsider items to improve the reliability of this scale.
This study also investigated gender and age-related differences associated with different bystander roles. The results show that boys report assisting the bully more often than girls, while girls report defending victims more often than boys. These results are partly in line with the Salmivalli et al. study (9) which found more girls than boys in the roles of defender and outsider, and more boys than girls in the roles of bully, assistant and reinforcer. Similarly, Thornberg and Jungert (34) found that boys' self-ratings of pro-bully behaviour were significantly higher when compared to girls, while girls were significantly more prone than boys to act as defenders and outsiders. In our study, gender differences in frequency of involvement in the reinforcer role, as well as in the aggressive defender role, were not significant. Gender differences in assisting the bully and defending the victim might be explained in terms of gender-specific socialization in which girls are more encouraged to engage in nurturing and caring behaviours, whereas aggression is more socially acceptable for boys $(13,38)$. This is in line with the results of meta-analyses which have found that boys are more inclined than girls to use direct aggression (39) and they also engage more in bullying actions than girls (40). Non-significant gender differences on the Reinforcer scale, compared to the Assistant scale, could be explained by differences in the types of aggressive behaviour these scales assess. Reinforcer items describe passive involvement in bullying and covert aggression which is more typical for girls' behaviour in bullying situations, while assistant items include direct forms of bullying, which are more typical for boys than girls. The age of the participants was significantly positively associated with reinforcing the bully and negatively associated with defending the victim. This is in line with findings showing that, as children get older, they are increasingly more likely to reinforce the bully (14) and less 
likely to defend the victimized peers (17). In terms of gender differences in emotional empathy and moral disengagement, the results of this study confirm the findings of other studies that girls have higher mean scores on self-report measures of affective empathy than boys (38), while boys have higher mean scores in moral disengagement than girls (29, $34,41)$.

The main aim of the present study was to examine whether moral disengagement and empathy uniquely contributed to the explanation of each of the bystander roles in bullying situations. Students' capacity to experience higher emotional empathy was related to a lower tendency to activate the moral disengagement mechanism. This is in line with previous studies showing that affective empathy (33) and moral emotions (an index of empathy, sympathy and guilt) are negatively related to moral disengagement (41). The results of hierarchical regression analyses revealed that emotional empathy was the variable most strongly related to the variance in the defender scores, showing a positive independent effect on defender behaviour, while moral disengagement had a smaller independent negative effect on the defender role. Moral disengagement was the strongest independent predictor of assistant and reinforcer scores, showing positive associations with these roles. Empathy had a small, but significant, independent negative effect on the reinforcer role. Overall, these results are consistent with previous meta-analytic findings showing a positive association between affective empathy and defenders' behaviour (40) and a positive association between moral disengagement and aggressive behaviour, including bullying (30). The results on the relationship between empathy and the reinforcer role partly confirm the findings that children and adolescents with a low level of empathy are more likely to display pro-bullying behaviour $(25,26)$. In line with previous studies, the results of the current study show that students with higher levels of moral disengagement are more likely to take assistant and reinforcer roles, i.e. pro-bullying roles (31, 34 ), and less likely to take the defender role $(34,41)$. While moral disengagement was a significant negative predictor of the defender role, this variable showed an independent positive effect on aggressive defender behaviour. Empathy was also positively associated with defending the victim in aggressive ways, acting as a supressor variable in a regression analysis. Aggressive defenders show reactive aggression directed towards the bully and, similarly to pro-bully roles, they are prone to activating mechanisms of moral disengagement to justify their behaviour. Their tendency to experience emotional empathy and defend the victim might result from sharing similar experience as victims of school bullying. Salmivalli et al. (9) found that victims scored significantly higher on the Defender Scale, compared to non-victimised children. These researchers also identified a small group of controversial children, i.e. children who received above average acceptance and rejection scores in their peer group. Controversial children had above average scores on pro-bullying and defending scales.

The results of regression analyses confirmed independent effects of students' gender and age on some bystander roles. Older age of the participants predicted higher involvement in reinforcing the bully and lower involvement in defending the victim. Boys were more frequently involved in assisting the bully than girls. Gender differences in defending the victim were not significant when differences in emotional empathy and moral disengagement between girls and boys were controlled.

It should be noted that students' individual characteristics explained a relatively small proportion of variance in bystanders' behaviours, indicating that other important factors 
that were not included in this study might be related to different bystander roles. For example, research has revealed that it is important to examine bystanders' responses to bullying at the classroom level. Bullying occurs more frequently in classrooms with higher levels of reinforcing the bully and lower levels of defending the victim $(42,43)$. Future research may need to examine other personality and socio-cognitive characteristics of bystanders of school bullying and classroom dynamics in which their behaviour occurs. In addition, the stability of the factor structure of the adapted PRQ scales in other samples should be confirmed.

From the point of view of prevention, the present findings suggest that it is important to develop activities and programmes aimed at enhancing bystanders' affective empathy and decreasing their moral disengagement in order to encourage more students to intervene on behalf of victims of bullying. The behaviour of the bullies might be difficult to change if the peer context is ignored. It can be more easily changed by influencing the behaviour of the classmates. In this way, social rewards that are important to bullies could be reduced, which could lead to a reduction in bullying (2). Anti-bullying prevention and intervention programmes applied in the classroom should emphasise raising adolescents' awareness of the role they play as witnesses of bullying situations, empathy training, especially the ability to share others' emotions, and emotional skills development (23, 33). Prevention should also raise bystanders' awareness of their moral transgressions and develop strategies for inhibiting moral disengagement mechanisms through efforts to enhance empathy for victimised peers $(30,43)$.

\section{Limitations of the Study}

There are also some limitations of this study which need to be addressed. First, due to the cross-sectional methodology of the study we cannot make any conclusions about the causal and temporal relationships between the studied variables. For example, it is not clear whether moral disengagement is a predictor of pro-bullying behaviour or serves as an excuse to justify prior aggression. It is also possible that the relations found in the current study are reciprocal. Longitudinal studies are needed to adequately examine the direction of the effects. Second, our sample included students attending higher elementary school grades (fifth to eighth) from two schools in Croatia. Further studies should include a wider age range and a larger, more heterogenous sample of adolescents. Finally, the measures of bystander behaviours were obtained through students' self-reports. Future research may employ multiple methods of data collection (e.g. peer-nominations, observation) and multiple informants (e.g. peers, teachers) to confirm the validity of students' reports.

\section{Conclusion}

The present study fills the gap in the literature by exploring the simultaneous effects of affective empathy and moral disengagement on various bystander roles and showing how these individual characteristics inhibit or reinforce pro-bully or pro-victim behaviour. Our findings demonstrate the validity of differentiating between assertive and aggressive defender roles when assessing participant roles in bullying situations. Moral disengagement mechanisms seem to play an important role in enhancing students' behaviour in assistant (of the bully), reinforcer (of the bully), and aggressive defender (of the victim) roles, as well as in inhibiting defending the victim in assertive ways. Emotional empathy plays an important role in enhancing defending the victim in assertive ways. Empathy is also a significant positive predictor of aggressive 
defender behaviour and a negative predictor of reinforcer behaviour. Efforts to counter school bullying should include intervention approaches aimed at students who witness bullying episodes, particularly at enhancing their empathic responsiveness towards victimised peers and inhibiting moral disengagement mechanisms that justify aggressive behaviour.

Authors' Contributions: Conception and design: ZRŠ, SB; Acquisition, analysis and interpretation of data: ZRŠ, SB; Drafting the article: ZRŠS, SB; Revising it critically for important intellectual content: ZR $\check{S}$; Approved final version of the manuscript: ZRŠ, SB.

Conflict of Interest: The authors declare that they have no conflict of interest.

\section{References}

1. Olweus D. Bullying at school: What we know and what we can do [in Croatian]. Zagreb: Školska knjiga; 1998.

2. Salmivalli, C. Bullying and the peer group: A review. Aggress Violent Behav. 2010;15(2):112-20.

3. Shetgiri R. Bullying and victimization among children. Adv Pediatr. 2013;60(1):33-51.

4. Moore SE, Norman RE, Sly PD, Whitehouse AJ, Zubrick SR, Scott J. Adolescent peer aggression and its association with mental health and substance use in an Australian cohort. J Adolesc. 2014;37(1):11-21.

5. Gini G, Pozzoli T. Association between bullying and psychosomatic problems: A meta-analysis. Pediatrics 2009;123(3);1059-65.

6. Kim YS, Leventhal B. (2008). Bullying and suicide. A review. Int $\mathrm{J}$ Adolesc Med Health. 2008;20(2):133-54.

7. Sesar K, Sesar D. Bullying behaviour in relation to psychological difficulties: Prospective research [in Croatian]. Drus Istraz. 2013;22(1):79-100.

8. Farrington DP, Ttofi MM. Bullying as a predictor of offending, violence and later life outcomes. Crim Behav Mental Health. 2011;2(2):90-8.

9. Salmivalli C, Lagerspetz K, Björkqvist K, Österman K, Kaukiainen A. Bullying as a group process: Participant roles and their relations to social status within the group. Aggr Behav. 1996;22(1):1-15.
10. O’Connell P, Pepler D, Craig W. Peer involvement in bullying: Insights and challenges for intervention. J Adolesc. 1999;22(4):437-52.

11. Meter DJ, Card NA. Defenders of victims of peer aggression: Interdependence theory and an exploration of individual, interpersonal, and contextual effects on the defender participant role. Dev Rev. 2015;38:222-40.

12. Sainio M, Veenstra R, Huitsing G, Salmivalli C. Victims and their defenders: A dyadic approach. Int J of Behav Dev. 2010;35(2):144-151.

13. Goossens FA, Olthof T, Dekker PH. New participant role scales: Comparison between various criteria for assigning roles and indications for their validity. Aggr Behav. 2006;32(4):343-57.

14. Salmivalli C, Voeten M. Connections between attitudes, group norms, and behaviour in bullying situations. Int J of Behav Dev. 2004; 28(3):246-58.

15. Sutton J, Smith PK. Bullying as a group process: An adaptation of the participant role approach. Aggr Behav. 1999;25(2):97-111.

16. Velki T, Kuterovac Jagodić G. Different measurement approaches as sources of differences in data on the prevalence of peer violence. Drus Istraz. 2014;23(2):259-81.

17. Pöyhönen V, Juvonen J, Salmivalli C. What does it take to stand up for the victim of bullying?: The interplay between personal and social factors. Merrill Palmer Q. 2010;56(2):143-63.

18. Tani F, Greenman PS, Schneider BH, Fregoso M. A Study of childhood personality and participant roles in bullying incidents School Psychol Int. 2003;24(2):131-46.

19. Strayer J. Affective and cognitive perspectives on empathy. In: Eisenberg N, Strayer J, editors. Empathy and its development. New York: Cambridge University Press; 1987. p. 218-44.

20. Eisenberg N, Miller P. The relation of empathy to prosocial and related behaviors. Psychol Bull. 1987;101(1):91-119.

21. Miller PA, Eisenberg N. The relationship of empathy to aggressive and externalizing/antisocial behavior. Psychol Bull. 1988;103(3):324-44.

22. Dodaj A, Sesar K, Barišić M, Pandža M. The effect of empathy on involving in bullying behavior. Paediatrics Today. (2013);9(1);91-101.

23. van Noorden TH, Haselager GJ, Cillessen AH, Bukowski WM. Empathy and involvement in bul- 
lying in children and adolescents: A systematic review. J Youth Adolesc. 2015;44(3):637-57.

24. Lucas-Molina B, Williamson AA, Pulido R, Calderón S. Adaptation of the Participant Role Scale (PRS) in a Spanish youth sample: Measurement invariance across gender and relationship with sociometric status. J Interpers Violence. 2014;29(16);2904-30.

25. Gini G, Albiero P, Benelli B, Altoè G. Does empathy predict adolescents' bullying and defending behavior? Aggr Behav. 2007;33(5);467-76.

26. Demaray MK, Summers KH, Jenkins LN, Becker LD. Bullying Participant Behaviors Questionnaire (BPBQ): Establishing a reliable and valid measure. J Sch Violence. 2016;15(2):158-88.

27. Bilić V. Violence among peers in the real and virtual world. Paediatrics Today. 2013;9(1):78-90

28. Bandura A. Moral disengagement in the perpetration of inhumanities. Pers Soc Psychol Rev. 1999;3(3):193-209.

29. Bandura A, Barbaranelli C, Caprara GV, Pastorelli C. Mechanisms of moral disengagement in the exercise of moral agency. J Pers Soc Psychol. 1996;71(2):364-74.

30. Gini G, Pozzoli T, Hymel S. Moral disengagement among children and youth: A meta-analytic review of links to aggressive behavior. Aggr Behav. 2014;40(1):56-68.

31. Gini G. Social cognition and moral cognition in bullying: What's wrong? Aggr Behav. 2006;32(6), 528-39.

32. Hymel S, Rocke-Henderson N, Bonanno RA. Moral disengagement: A framework for understanding bullying among adolescents. J Soc Sci. 2005;8(1):1-11.

33. Kokkinos CM, Kipritsi E. Bullying, moral disengagement and empathy: Exploring the links among early adolescents. Educ Psychol. 2018;38(4):535-52.

34. Thornberg R, Jungert T. Bystander behavior in bullying situations: Basic moral sensitivity, moral dis- engagement and defender self-efficacy. J Adolesc. 2013;36(3):475-83.

35. Raboteg-Šarić Z. (2002). E-Questionnaire: The Emotional Empathy Scale and Fantasy Scale [in Croatian]. In: Lacković-Grgin K, Proroković A, Ćubela V, Penezić Z, editors. Zbirka psihologijskih skala i upitnika. Zadar: Filozofski fakultet u Zadru; 2002. p. 44-9.

36. Hawkins DL, Pepler DJ, Craig WM. Naturalistic observations of peer interventions in bullying. Soc Dev. 2001;10(4):512-27.

37. Pozzoli T, Gini G. (2010). Active defending and passive bystanding behavior in bullying: The role of personal characteristics and perceived peer pressure. J Abnorm Child Psychol. 2010;38(6):815-27.

38. Eisenberg N, Lennon R. Sex differences in empathy and related capacities. Psychol Bull. 1983;94(1):100-31

39. Card NA, Stucky BD, Sawalani GM, Little TD. Direct and indirect aggression during childhood and adolescence: A meta-analytical review of gender differences, intercorrelations, and relations to maladjustment. Child Dev. 2008;79(5):1185-229.

40. Mitsopoulou E, Giovazolias T. Personality traits, empathy and bullying behavior: A meta-analytic approach. Aggress Violent Behav. 2015;21:61-72.

41. Thornberg R, Pozzoli T, Gini G, Jungert, T. Unique and interactive effects of moral emotions and moral disengagement on bullying and defending among school children. Elem Sch J. 2015;116(2):322-37.

42. Salmivalli C, Voeten M, Poskiparta E. Bystanders matter: Associations between reinforcing, defending, and the frequency of bullying behavior in classrooms. J Clin Child and Adolesc Psychol. 2011;40(5):668-76.

43. Thornberg R, Wänström L. Bullying and its association with altruism toward victims, blaming the victims, and classroom prevalence of bystander behaviors: A multilevel analysis. Soc Psychol Educ. 2018;21(5):1133-51. 